\title{
Efecto analgésico de la compresión isquémica con billas de acero inoxidable en pacientes con discapacidad cervical
}

\section{Paul Wong-Lizano'}

RESUMEN: La cervicalgia es un problema de salud importante y una de las causas de discapacidad que generan limitaciones en la realización de las actividades cotidianas. Los centros de salud en el Perú brindan tratamientos alternativos de compresión isquémica. Sin embargo, la eficacia de la compresión isquémica con billas de acero inoxidable no ha sido aun suficientemente comprobada. Por ello, el objetivo fue determinar la existencia del efecto analgésico usando la compresión isquémica con billas de acero inoxidable en pacientes con discapacidad cervical. Se aplicaron las billas de acero en los puntos dolorosos en la zona cervical, tres veces por semana, de forma continua durante 25 minutos por sesión a 50 pacientes. Se incluyeron pacientes que tenían discapacidad cervical leve y moderada. Se comprobó una diferencia de 5.7 puntos en la escala visual analógica entre el promedio de la primera evaluación (8.26) y la final (2.56), siendo este resultado estadísticamente significativo $(\mathrm{p}<0.001)$.

PALABRAS CLAVE: Discapacidad; Dolor cervical; Terapias alternativas. $\begin{array}{ll}\text { Citar como: } & \text { Wong P. Efecto analgésico de la compresión isquémica con billas de acero } \\ & \text { inoxidable en pacientes con discapacidad cervical. CASUS. 2017;2(3):163- }\end{array}$ 168. 


\section{INTRODUCCIÓN}

La cervicalgia es un problema a nivel global y una de las causas de discapacidad en las personas. Así mismo, es definida como el dolor situado en el área cervical o cuello $(1,2)$. Por otra parte, la discapacidad se encuentra vinculada a las diferentes deficiencias, limitaciones y restricciones de las actividades como consecuencia de una afección en el organismo $(3,4)$.

Entre un $30 \%$ y $50 \%$ de la población general padece de cervicalgia anualmente. Se observa también que entre el $11 \%$ y $14 \%$ de la población laboral la padece y un $15 \%$ presenta dolor crónico en la zona cervical lo cual genera una reducción en su capacidad productiva $(5,6)$. Esto predispone a presentar un mayor grado de discapacidad y de consecuencia dificultad en realizar sus labores (7). Por lo tanto, la cervicalgia origina una deficiencia en la estructura del cuello, lo cual conlleva a una limitación de la realización de actividades cotidianas (7). Esta situación, a su vez, puede provocar en el sujeto afectaciones en el desempeño del rol social que le es propio (4).

Entre los tratamientos más usados para el alivio de la cervicalgia se encuentra el tratamiento fisioterapéutico que consiste en la administración de técnicas manuales y ejercicios terapéuticos $(8,9)$. La acupuntura, que se basa en la aplicación de agujas muy finas de acero inoxidable sobre canales o meridianos para regular el flujo de energía sobre el punto doloroso $(1,10)$. Los masajes que se administran sobre la zona dolorosa y el roce de las manos sobre la piel varían según la intensidad con que se apliquen. Por último, la digitopuntura o compresión isquémica como se le conoce en la actualidad. La misma consta de la presión mantenida con la yema de los dedos sobre un punto doloroso (11).

Actualmente en los centros de salud del Estado se está brindando el tratamiento de terapias alternativas donde es posible encontrar acupuntura, digitopuntura o compresión isquémica. Estos tratamientos se ofrecen como complemento a los tratamientos médicos o fisioterapéuticos. Sin embargo, la eficacia de la compresión isquémica con billas de acero inoxidable no ha sido aún suficientemente comprobada, menos aún en personas con discapacidad cervical. Por esa razón el objetivo del estudio fue determinar el efecto analgésico usando la compresión isquémica con billas de acero inoxidable en pacientes con discapacidad cervical.

\section{MATERIALES Y MÉTODOS}

El estudio fue explicativo con diseño preexperimental. La muestra estuvo conformada por 50 pacientes con discapacidad cervical leve a moderada en un centro de terapia de Lima. El muestreo fue no probabilístico de tipo intencional. Se incluyeron pacientes que tenían discapacidad cervical leve y moderada medida por la Escala de Discapacidad Cervical y que realizaban alguna actividad laboral o estudiantil (12). Se excluyeron a las personas que estaban tomando algún medicamento con efecto analgésico, diagnosticados con alguna enfermedad mental, alguna presencia de fracturas, alteraciones morfológicas o cáncer.

La variable dependiente cervicalgia mecánica se midió a través de la Escala Visual Análoga del Dolor (EVA), variable cuantitativa entre un rango de cero (dolor mínimo o ausencia del dolor) a diez (dolor máximo).

Para la intervención se procedió primeramente con la evaluación inicial del dolor. Posteriormente, se aplicaron las billas de acero inoxidables en los puntos dolorosos en la zona cervical, tres veces por semana, de forma continua durante un tiempo de 25 minutos por sesión. El tiempo de intervención fue de cinco semanas. Para ello se le pidió al paciente descubrir el área del cervical y adoptar una posición de decúbito prono sobre la camilla. Luego se procedió a colocar las billas de acero de $0.5 \mathrm{~cm}$ haciendo presión sobre el punto doloroso y se fijaron haciendo uso de un esparadrapo antialérgico (ver figura 1). Terminada la sesión se procedió al retiro de las billas de acero inoxidable y a la reevaluación del dolor del paciente, mediante EVA.

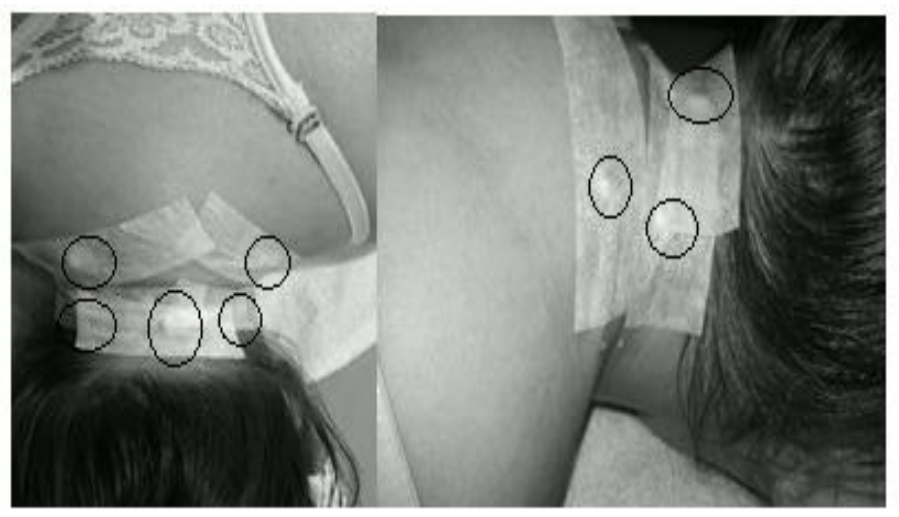

Figura 1. Aplicación de las billas de acero en la zona cervical 
Respecto a las variables sociodemográficas fueron consideradas el sexo: variable categórica (masculino, femenino); estado civil: variable categórica (soltero, casado, viudo, conviviente); situación laboral: variable categórica (trabaja, no trabaja); antecedentes de cervicalgia: variable categórica (sí, no) y la práctica habitual de ejercicios: variable categórica (sí, no).

Para la realización del análisis estadístico se utilizó el programa Stata versión 12. En el análisis descriptivo de las variables categóricas se emplearon frecuencias y porcentajes. Para el nivel de dolor se emplearon medias y desviaciones estándares. Se realizó la prueba de normalidad de Shapiro Wilk para la variable numérica. Se utilizó la prueba de T-Student para muestras pareadas para determinar la eficacia de la intervención en la cervicalgia mecánica. De igual modo, se realizó un cálculo de potencia estadística para determinar si el tamaño muestral era óptimo. Finalmente se determinó una significancia estadística menor a 0.05 .

El estudio contó con la aprobación del comité de ética institucional de la Universidad Católica Sedes Sapientiae, asimismo se obtuvo el consentimiento informado a la participación al estudio respetándose la privacidad y la confiabilidad de los datos de los pacientes.

\section{RESULTADOS}

Se observó entre los pacientes una mayor cantidad de mujeres (56\%). Del total el 52\% eran casados y el $62 \%$ trabajaba al momento del estudio. Además, el $76 \%$ no tenía antecedentes de cervicalgia. Finalmente, el 58\% no realizaba alguna actividad física en forma rutinaria (ver Tabla 1 ).

Se comprobó una diferencia de 5.7 puntos en EVA entre el promedio de la primera evaluación (8.26) y la final (2.56), siendo este resultado estadísticamente significativo $(\mathrm{p}<0.001)$. También se pudo observar un resultado similar en las evaluaciones de seguimiento que se realizaron semanalmente, exceptuando la última donde no se encontró diferencia significativa entre las mediciones. Se observó una disminución del dolor durante el transcurso del tratamiento hasta la cuarta semana. En la misma, la media del dolor percibido llegó a una puntuación de 2.58 en la escala EVA. Está puntuación se mantuvo hasta el final de las sesiones (ver Tabla 2).
Tabla1. Descripción de las variables sociodemográficas

\begin{tabular}{cc}
\hline \multicolumn{1}{c}{ Variables } & $\mathbf{n}(\%)$ \\
\hline Sexo & \\
Masculino & $22(44)$ \\
Femenino & $28(56)$ \\
Estado civil & \\
Soltero & $18(36)$ \\
Casado & $26(52)$ \\
Viudo & $4(8)$ \\
Conviviente & $2(4)$ \\
Situación laboral & $31(62)$ \\
Trabaja & $19(38)$ \\
No trabaja & \\
Antecedentes de \\
cervicalgia \\
Sí \\
No \\
Practica ejercicios \\
habitualmente \\
Sí \\
No \\
\hline
\end{tabular}

Tabla 2. Promedios del EVA inicial y final

\begin{tabular}{lcccc}
\hline & Inicial & Final & Delta $^{*}$ & $\mathbf{p}^{\star \star}$ \\
\hline $\begin{array}{l}\text { Evaluación } \\
\text { del dolor }\end{array}$ & $8.26 \pm 1.15$ & $2.56 \pm 1.35$ & $5.7 \pm 1.38$ & $<0.001$ \\
$\begin{array}{l}\text { 1era } \\
\text { semana }\end{array}$ & $8.26 \pm 1.15$ & $6.02 \pm 1.30$ & $2.24 \pm 1.11$ & $<0.001$ \\
2da semana & $5.64 \pm 1.13$ & $4.90 \pm 1.29$ & $0.74 \pm 0.85$ & $<0.001$ \\
3era & $4.06 \pm 1.54$ & $3.22 \pm 1.43$ & $0.84 \pm 1.03$ & $<0.001$ \\
semana & & & & \\
4ta semana & $3.72 \pm 0.70$ & $2.58 \pm 1.32$ & $1.14 \pm 1.19$ & $<0.001$ \\
5ta semana & $2.54 \pm 1.29$ & $2.56 \pm 1.35$ & $-0.02 \pm$ & 0.811 \\
& & & -0.18 & \\
& & & &
\end{tabular}

*Diferencia entre el EVA inicial y final.

${ }^{* *}$ Corresponde a la prueba estadística T de Student para muestras relacionadas.

Se observó en los resultados obtenidos que entre los grupos el beneficio de la aplicación es similar, a diferencia de los grupos de situación laboral. Se observó que las personas que trabajaban presentaron un mayor beneficio de la aplicación de la técnica comparados con los que no trabajaban (6.06 y 5.10). Los hombres presentaron mayor beneficio que las mujeres (5.9 y 5.6). Para ambos casos el resultado fue estadísticamente significativo ( $\mathrm{p}<0.001)$ (ver tabla 3$)$.

\section{DISCUSIÓN}

El estudio se ejecutó en un periodo de cinco semanas realizándose una evaluación inicial y otra final del dolor. También se tomaron controles por semana y se obtuvo que el dolor disminuyó hasta 2.58 puntos EVA. 
Tabla 3. Efecto del tratamiento con relación a las variables sociodemográficas

\begin{tabular}{|c|c|c|c|c|}
\hline \multirow{2}{*}{\begin{tabular}{l}
\multicolumn{1}{c}{ Variables } \\
Evaluación del dolor \\
Datos sociodemográficos \\
Sexo
\end{tabular}} & EVA $\mathbf{i}$ & EVAf & Delta* ${ }^{*}$ & $\mathbf{p}^{* *}$ \\
\hline & $8.26 \pm 1.15$ & $2.56 \pm 1.35$ & $5.7 \pm 1.38$ & $<0.001$ \\
\hline Masculino & $8.22 \pm 1.10$ & $2.36 \pm 1.17$ & $5.86 \pm 1.35$ & $<0.001$ \\
\hline Femenino & $8.28 \pm 1.21$ & $2.71 \pm 1.48$ & $5.57 \pm 1.42$ & $<0.001$ \\
\hline Situación laboral & & & & \\
\hline Trabaja & $8.42 \pm 1.06$ & $2.41 \pm 1.17$ & $6.06 \pm 1.18$ & $<0.001$ \\
\hline No trabaja & $7.89 \pm 1.24$ & $2.78 \pm 1.61$ & $5.10 \pm 1.53$ & $<0.001$ \\
\hline Antecedente de cervicalgia & & & & \\
\hline Sí & $8.33 \pm 1.15$ & $2.58 \pm 1.16$ & $5.75 \pm 1.28$ & $<0.001$ \\
\hline No & $8.23 \pm 1.17$ & $2.55 \pm 2.08$ & $5.68 \pm 1.43$ & $<0.001$ \\
\hline Practica ejercicios habitual & & & & \\
\hline Sí & $8.19 \pm 1.28$ & $2.71 \pm 1.41$ & $5.47 \pm 4.71$ & $<0.001$ \\
\hline No & $8.31 \pm 1.07$ & $2.44 \pm 1.32$ & $5.86 \pm 5.42$ & $<0.001$ \\
\hline
\end{tabular}

*Diferencia entre el EVA inicial (EVA i) y EVA final (EVA f).

${ }^{* *}$ Corresponde a la prueba estadística T de Student.

Esto se debe a la respuesta fisiológica que ocurre al realizar una isquemia localizada en un punto doloroso. Lo anterior ayuda a la reabsorción del material de desecho, mejora la vascularización en el tejido y la musculatura se relaja (13). Igualmente, los niveles de dolor disminuyen por el estímulo sobre los receptores de tacto y presión a nivel de piel y músculo (14).

Se observó que los hombres presentaron un mayor beneficio frente a las mujeres en la disminución del dolor. Un estudio reporta diferencias respecto al sexo en lograr analgesia, indicando que las mujeres reportan niveles más altos en la intensidad del dolor. Por ello, requirieron mayor cantidad de analgésico para lograr niveles similares de alivio del dolor respecto a los hombres (15).

De igual modo las personas que trabajan reportaron beneficios superiores en la disminución del dolor. Esta diferencia se debería a que el grupo de personas que labora es del $62 \%$ en comparación al que no labora $48 \%$. Otro punto importante es que en el grupo de personas que no laboran se encuentran las amas de casa quienes presentan el mayor porcentaje de dolor cervical frente a personas que realizan otras actividades, ya sean de ocio o laborales (16).

Dentro de las limitaciones del estudio se menciona la posibilidad de sesgo de selección, así como el tamaño reducido de la muestra del estudio. Por otro lado, no se contó con un grupo control para contrastar el efecto en los pacientes del grupo experimental frente a la terapia física convencional. Sin embargo, el estudio es novedoso aportando evidencia sobre el efecto analgésico del uso de billas de acero sobre puntos dolorosos en el área cervical.

Se debe tener en cuenta que los fisioterapeutas, además, deberían emplear más de un enfoque terapéutico para el tratamiento de pacientes con cervicalgia y no deberían usar únicamente la compresión isquémica con billas de acero como intervención aislada. Se sugiere que los estudios futuros investiguen si esta técnica mejora los resultados cuando se agrega a las intervenciones con eficacia comprobada, como el ejercicio activo (18). Igualmente, se recomienda se tomen en cuenta un número mayor de pacientes y realizar comparaciones frente a otros tratamientos, incrementar el número de días de tratamiento para un mejor control del dolor. Del mismo modo, se deben detallar las actividades laborales de los pacientes, distribuir a los pacientes por rango de edades indagando sobre las posturas forzadas ya sea en el trabajo o en otras actividades.

\section{CONCLUSIÓN}

Se comprobó que existe un efecto analgésico en la aplicación de la compresión isquémica con billas de acero en pacientes con cervicalgia mecánica con un efecto semanal mantenido. De igual modo, en los hombres en los que trabajan existe mayor efecto reductor sobre el nivel de dolor. Basados en los resultados de este estudio se podría implementar el uso de la compresión isquémica con billas de acero como un tratamiento alternativo o complementario no invasivo y de bajo costo al tratamiento de cervicalgias en el área de fisioterapia. 


\section{REFERENCIAS BIBLIOGRÁFICAS}

1. Rodríguez L, Valdés Y. Evolución clínica de los pacientes con cefalea migrañosa tratados con digitopuntura. Revista Cubana Habanera de Ciencias Médicas. 2010;9(1)27-38.

2. Cousins M. International association for the study of pain. Pain. 1989;39(2);1-8.

3. Organización Mundial de la Salud. Discapacidades [internet]. Ginebra, Suiza: OMS; 2017. [citado 10 nov 2017]. Disponible en: http://www.who.int/topics/disabilities/es/

4. Villanueva C, Leyba J, Peralta V. Las clasificaciones de la enfermedad y la discapacidad de la OMS. Fisioterapia. 2005;27(5):274-83. doi: 10.1016/S02115638(05) $73450-7$

5. Kazemi A, Muñoz L, Martín J, Pérez M, Henche M. Estudio etiopatogénico de la cervicalgia en la población general basado en la exploración física. Revista de la Sociedad Española del Dolor. 2000;7(4):220-224.

6. Fejer R, Kyvik K, Hartvigsen J. The prevalence of neck pain in the world population: a systematic critical review of the literature. European Spine Journal. 2006;15(6), pp.834-848. doi: 10.1007/s00586-004-0864-4

7. Fry H. The treatment of overuse syndrome in musicians. Results in 175 patients. Journal of Royal Society of Medicine. 1988;81(10):57257. PubMed Central PMCID: PMC1291799.

8. Pérez Y, Díaz B, Lebrijo G. Efectividad del tratamiento fisioterápico en pacientes con cervicalgia mecánica. Fisioterapia. 2002; 24(3):165-174.

9. Arce M. Eficacia de tres tratamientos rehabilitadores en la lumbalgia crónica por síndrome miofascial del cuadrado lumbar, en pacientes del Hospital Luis Heysen Inchaustegui [tesis]. Chiclayo: Universidad San Martín de Porres. Facultad de Medicina; 2013.

10. Laitinen J. Treatment of cervical syndrome by acupuncture. Pain. 1977;3(3):284- 285.

11. González J, Fernández C, Cleland JA, Huijbregts P , Del Rosario M. Short-Term Effects of Cervical Kinesio Taping on Pain and Cervical Range of Motion in Patients With Acute Whiplash Injury: A Randomized Clinical Trial. J Orthop Sports Phys Ther.
2009;39(7):515-521. doi:10.2519/jospt.2009.3072.

12. Chaitow L, Fritz S. Cómo conocer, localizar y tratar los puntos gatillo miofasciales. 1a ed. Barcelona: Elsevier;2008.

13. Yamauchi G, Cheng A. La digitopuntura es eficaz para tratar pacientes con lumbalgia crónica. Revista Evidencia. 2006;9(4):108.

14. Travell J, Simons D. Myofascial pain and dysfunction: The trigger point manual. The lower extremities. 2a ed. Philadelphia: Lippincott Williams \& Wilkins; 1992.

15. Kest B, Sarton E, Dahan A. Gender differences in opioid mediated analgesia: animal and human studies. Anesthesiology. 2000;93(2):539-547.

16. Cepeda MS, Carr DB. Women experience more pain and require more morphine than men to achieve a similar degree of analgesia. Anesthesia \& Analgesia. 2003;97(5):1464-8.

17. Bueno IS, Gómez CM, Roldán OV, Pons AA. Terapia manual y terapia combinada en el abordaje de puntos gatillo: revisión bibliográfica. Fisioterapia. 2009;31(1):17-23. 
ISSN 2519-0652 CASUS.2017;2(3):163-168

\section{Analgesic effect of ischemic compression with stainless steel spheres in patients with cervical disability}

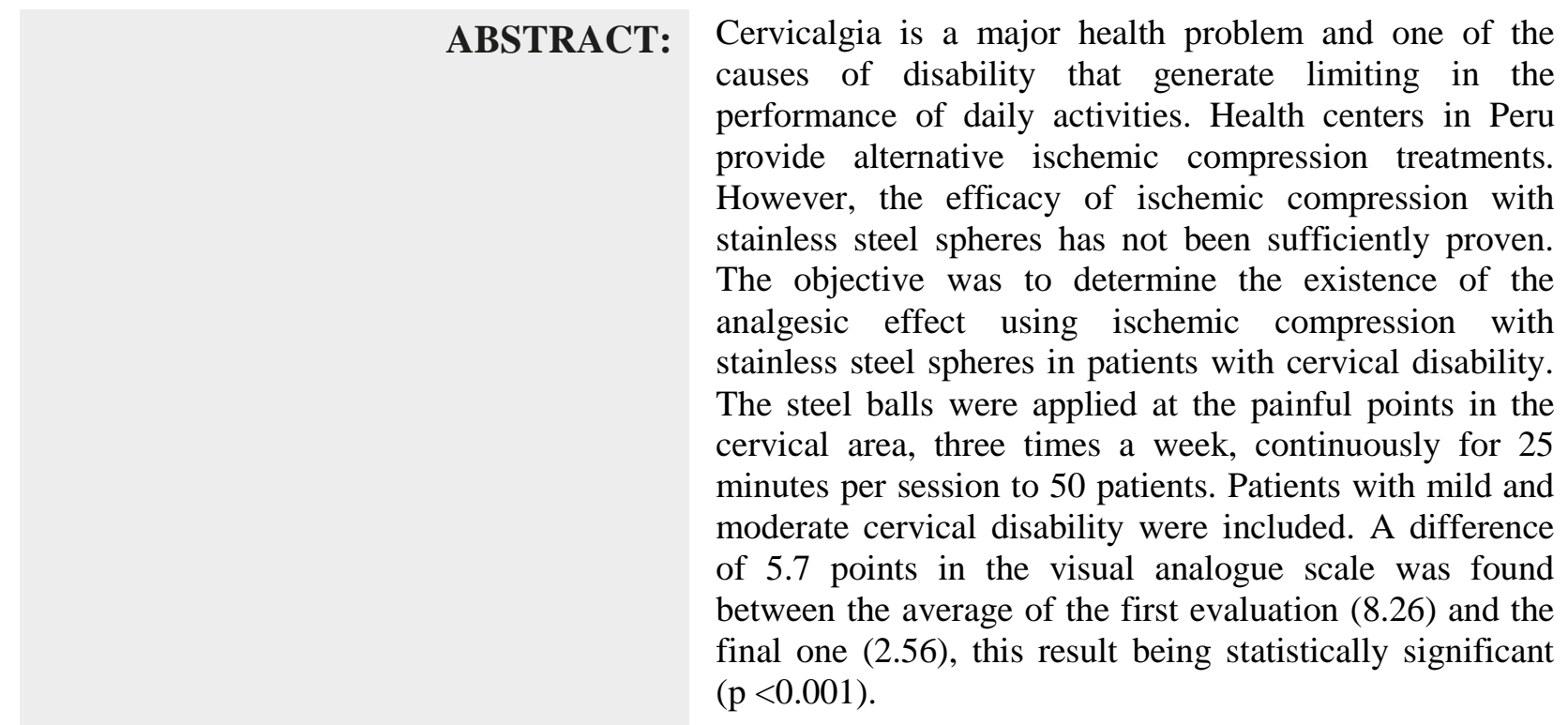

KEY WORDS:

Disability; Neck pain; Complementary Therapies. 